\title{
A Positive Answer for 3IM+1CM Problem with a General Difference Polynomial
}

\author{
Huicai Xu (D), ${ }^{1,2}$ Shugui Kang, ${ }^{2}$ and Qingcai Zhang $\mathbb{D}^{1}$ \\ ${ }^{1}$ School of Mathematics, Renmin University of China, Beijing 100872, China \\ ${ }^{2}$ School of Mathematics and Statistics, Shanxi Datong University, Datong 037009, China \\ Correspondence should be addressed to Qingcai Zhang; zhangqcrd@163.com
}

Received 28 September 2021; Accepted 5 November 2021; Published 27 November 2021

Academic Editor: Li Li

Copyright (c) 2021 Huicai Xu et al. This is an open access article distributed under the Creative Commons Attribution License, which permits unrestricted use, distribution, and reproduction in any medium, provided the original work is properly cited.

In this paper, the $3 \mathrm{IM}+1 \mathrm{CM}$ theorem with a general difference polynomial $L(z, f)$ will be established by using new methods and technologies. Note that the obtained result is valid when the sum of the coefficient of $L(z, f)$ is equal to zero or not. Thus, the theorem with the condition that the sum of the coefficient of $L(z, f)$ is equal to zero is also a good extension for recent results. However, it is new for the case that the sum of the coefficient of $L(z, f)$ is not equal to zero. In fact, the main difficulty of proof is also from this case, which causes the traditional theorem invalid. On the other hand, it is more interesting that the nonconstant finite-order meromorphic function $f$ can be exactly expressed for the case $f \equiv-L(z, f)$. Furthermore, the sharpness of our conditions and the existence of the main result are illustrated by examples. In particular, the main result is also valid for the discrete analytic functions.

\section{Introduction and Results}

It is well known that any polynomial is uniquely determined by its zero points (the set on which the polynomial takes zeros) except for a nonconstant factor, but it is not true for transcendental entire or meromorphic functions. For example, functions $e^{z}$ and $e^{-z}$ have the same $\pm 1,0$ and $\infty$ points. Thus, it is interesting and complex to uniquely determine a meromorphic function. It is well known that the classical uniqueness results of the value distribution theory of meromorphic functions are the 5IM theorem (or fivepoint theorem) and the 4CM theorem (or four-point theorem) which had been obtained in the study by Nevanlinna [1], where IM means ignoring multiplicities and CM is counting multiplicities.

Let $f$ and $g$ be two nonconstant meromorphic functions on the complex plane $\mathbb{C}$ and $a$ be a finite or infinite complex number in the whole complex plane $\widehat{\mathbb{C}}=\mathbb{C} \cup\{\infty\}$. If $f-a$ and $g-a$ have the same zeros with the same multiplicities, we say that $f$ and $g$ share $a$ CM (counting multiplicities). If not considering the multiplicities, we say that $f$ and $g$ share $a$ IM (ignoring multiplicities). Clearly, they also share $a$ IM if $f$ and $g$ share $a$ CM. In this case, the famous five-point theorem and four-points theorem mean that if two nonconstant meromorphic functions $f$ and $g$ share five distinct values IM, then $f=g$, and if two nonconstant meromorphic functions $f$ and $g$ share four distinct values CM, then $f$ is a factional linear transformation of $g$, respectively.

The 4CM theorem in the study by Nevanlinna [1] can also be understood to be the $4 \mathrm{CM}+0$ IM theorem which motivates us to consider the other cases as the $3 \mathrm{CM}+1 \mathrm{IM}$ theorem, the $2 \mathrm{CM}+2 \mathrm{IM}$ theorem, the $3 \mathrm{IM}+1 \mathrm{CM}$ theorem, or the $0 \mathrm{CM}+4 \mathrm{IM}$ theorem. Obviously, it is very good if the $0 \mathrm{CM}+4 \mathrm{IM}$ theorem is a fact. Unfortunately, Gundersen [2] has constructed counterexamples which show that the conclusion of the four-point theorem is invalid if CM is replaced by IM; that is, the $0 \mathrm{CM}+4 \mathrm{IM}$ theorem is false. However, the $2 \mathrm{CM}+2 \mathrm{IM}$ theorem is valid; please see the study by Gundersen [3]. It is known that the 2CM+2IM theorem implies that the $3 \mathrm{CM}+1 \mathrm{IM}$ theorem holds (see Gundersen [2, 3]). Thus, in [4], Gundersen proposed the following well-known question. 
Problem 1. (3IM+1CM open problem [4]). If two nonconstant meromorphic functions share three values IM and share a fourth value CM, then do the functions necessarily share all four values CM?

It is very difficult to completely solve Problem 1 (see Gundersen [4]). In the past years, some researchers established 3IM+1CM theorems with some conditions, for example, the restriction of zero points for $f-g$ (see Mues [5-7], Reinders [8, 9], Wang [10], Ueda [11, 12], Yi and Zhou [13], Czubiak and Gundersen [14], Qiu [15], Wang [16, 17], Huang [18, 19], Huang and Du [20], Ishizaki [21], Li [22], Yao [23], and Yang and Yi [24]), the periodicity hypothesis (see Lin and Ishizaki [25] and Banerjee and Ahamed [26]), or the differential limitation (see Lahiri and Sinha [27], Gundersen [28, 29], Mues and Steinmetz [30, 31], Mues and Reinders [32], and Frank and Hua [33]).

In recent years, the difference variant of the Nevanlinna theory has been established in [34-37]. Using these theories, some mathematicians began to consider the uniqueness of meromorphic functions sharing values with their shifts or difference operators and produced many fine works; for example, see Banerjee and Bhattacharyya [38], Ahamed [39, 40], Ma et al. [41], Jiang et al. [42], Charak et al. [43], Lin et al. [44, 45], and Li et al. [46, 47].

In this paper, we will establish the $3 \mathrm{IM}+1 \mathrm{CM}$ theorem with the difference condition. Actually, Li et al. [46] obtained the following uniqueness result when $f$ and $\Delta_{\eta}^{n} f$ fulfill the condition 3IM+1CM, where

$$
\begin{aligned}
& \Delta_{\eta} f(z)=f(z+\eta)-f(z), \\
& \Delta_{\eta}^{n} f(z)=\Delta_{\eta}\left(\Delta_{\eta}^{n-1} f(z)\right),
\end{aligned}
$$

for $n=2,3, \ldots$

Theorem 1 (see [46]). Let $f$ be a nonconstant meromorphic function with finite order, and let $\eta$ be a nonzero complex number. Suppose that $f$ and $\Delta_{\eta}^{n} f$ share $a_{1}, a_{2}, a_{3} I M$ and share $\infty C M$, where $a_{1}, a_{2}$, and $a_{3}$ are three distinct finite values. Then, $f(z)=\Delta_{\eta}^{n} f$ for all $z \in \mathbb{C}$.

Noting that

$$
\Delta_{\eta}^{n} f(z)=\sum_{i=0}^{n}\left(\begin{array}{l}
n \\
j
\end{array}\right)(-1)^{n-j} f(z+j \eta),
$$

naturally, a more general case

$$
L(z, f) \triangleq \sum_{j=0}^{n} \alpha_{j} f\left(z+d_{j}\right),
$$

should be considered, where $n \geq 1$ is a positive integer, $d_{j}(j=0, \ldots, n)$ are distinct finite values, and $\alpha_{j}(j=0, \ldots, n)$ are nonzero constants. In the case, Problem 1 turns to be the following problem.

Problem 2. When a nonconstant meromorphic function $f$ and its general linear difference polynomial $L(z, f)$ satisfy the condition $3 \mathrm{IM}+1 \mathrm{CM}$, does the uniqueness result still hold?

In the following, we will give our main result, some corollaries, and remarks.

Theorem 2. Let $f$ be a nonconstant meromorphic function with finite order; let $L(z, f)$ be defined as (3). If $f$ and $L(z, f)$ share $a_{1}, a_{2}, a_{3} I M$ and share $\infty C M$, where $a_{1}, a_{2}$, and $a_{3}$ are three distinct finite values, then for all $z \in \mathbb{C}$, one of the following results holds:

(i) $f=L(z, f)$;

(ii) $f=-L(z, f)$; furthermore,

$$
f(z)=c \frac{1+e^{a z+b}}{1-e^{a z+b}},
$$

and $\sum_{j=0}^{n} \alpha_{j}=-1$, where $c \in\left\{a_{1}, a_{2}, a_{3}\right\}$, and $a(\neq 0), b$ are constants.

To explain a nonconstant meromorphic function $f$ with finite order, we need the following definition.

Definition 1 (see $[24,48]$ ). For a nonconstant meromorphic function $f$, the order of $f$, denoted by $\rho(f)$, is defined as

$$
\rho(f)=\limsup _{r \longrightarrow \infty} \frac{\log T(r, f)}{\log r},
$$

where

$$
\begin{aligned}
& T(r, f)=m(r, f)+N(r, f), \\
& m(r, f)=\int_{0}^{2 \pi} \log ^{+}\left|f\left(r e^{i \theta}\right)\right| \mathrm{d} \theta, \\
& N(r, f)=\int_{0}^{r} \frac{n(t, f)-n(0, f)}{t} \mathrm{~d} t+n(0, f) \log r,
\end{aligned}
$$

and $n(t, f)$ denotes the number of poles of $f$ (counting multiplicities) in $|z|<t$.

A nonconstant meromorphic function $f$ with finite order means that $\rho(f)<\infty$.

Remark 1. The following example shows that Theorem 2 (ii) can happen. Let

$$
\begin{aligned}
f(z) & =a_{1} \frac{1+e^{a z+b}}{1-e^{a z+b}}, \\
L(z, f(z)) & =\sum_{j=0}^{n} \alpha_{j} a_{1} \frac{1+e^{a(z+j \eta)+b}}{1-e^{a(z-j \eta)+b}} \\
& =a_{1} \sum_{j=0}^{n} \alpha_{j} \frac{1+e^{a z+b}}{1-e^{a z+b}}=-a_{1} \frac{1+e^{a z+b}}{1-e^{a z+b}},
\end{aligned}
$$


where $a_{1} \neq 0, e^{a \eta}=1$, and $\alpha_{j}(j=0,1 \ldots, n)$ are nonzero constants satisfying $\sum_{j=0}^{n} \alpha_{j}=-1$, and then $f$ and $L(z, f)$ share $a_{1},-a_{1}, 0, \infty \mathrm{CM}$, and $f=-L(z, f)$.

Remark 2. The conditions of Theorem 2 are sharp. From the example in the following, we know that the assumption $3 \mathrm{IM}+1 \mathrm{CM}$ cannot be relaxed to $4 \mathrm{IM}$ in Theorem 2. For example, see Steinmetz [49]. Let $\wp$ denote the Weierstrass $\wp$-function with a pair of primitive periods $2 \omega-\omega^{\prime}$ and $\omega+\omega^{\prime}$, and set

$$
\begin{aligned}
f(z) & =\frac{(\wp(z)-\wp(\omega / 2))(\wp(z)-\wp(3 \omega / 2))^{2}}{\wp(z)-\wp(\omega)}, \\
L(z, f) & =f(z+\omega) .
\end{aligned}
$$

Then, the functions $f$ and $L(z, f)$ share the values $0, \infty$ and some values $A$ and $B \mathrm{IM}$, where $A / B=(1 / 2)(1 \pm \sqrt{3} i)$. We note that it is showed that three meromorphic functions $f(z), L(z, f)$, and $f\left(z+\omega^{\prime}\right)$ share the four values $0, \infty, A$, and $B$ IM in [49]. However, $f \neq L(z, f)$ or $f \neq-L(z, f)$.

Corollary 1. Let $f$ be a nonconstant meromorphic function with finite order; let $L(z, f)$ be defined as (3). Assume that $f$ and $L(z, f)$ share $a_{1}, a_{2}, a_{3} I M$ and share $\infty C M$, where $a_{1}, a_{2}, a_{3}$ are three distinct finite values. If $\sum_{j=0}^{n} \alpha_{j} \neq-1$, then $f=L(z, f)$ for all $z \in \mathbb{C}$.

Corollary 2. Let $f$ be a nonconstant meromorphic function with finite order; let $L(z, f)$ be defined as (3). Assume that $f$ and $L(z, f)$ share $a_{1}, a_{2}, a_{3} I M$ and share $\infty C M$, where $a_{1}, a_{2}, a_{3}$ are three distinct finite values. If $\sum_{j=0}^{n} \alpha_{j}=0$, then $f=L(z, f)$ for all $z \in \mathbb{C}$.

\section{Remark 3. Note that}

$$
\Delta_{\eta}^{n} f(z)=\sum_{i=0}^{n}\left(\begin{array}{c}
n \\
j
\end{array}\right)(-1)^{n-j} f(z+j \eta)
$$

and the sum of its coefficients $\sum_{i=0}^{n}\left(\begin{array}{c}n \\ j\end{array}\right)(-1)^{n-j}$ is zero. So, even if $\sum_{j=0}^{n} \alpha_{j}=0$ in $L(z, f), \Delta_{\eta}^{n} f(z)$ is still a special case of $L(z, f)$. Thus, Theorem 2 is a large extension of Theorem 1.2 in Li et al. [46]; naturally, the corresponding results in the study by Heittokangas et al. [50], Li et al. [46, 47], and so on are also the special cases of Theorem 2. However, our result is new when $\sum_{j=0}^{n} \alpha_{j} \neq 0$.

Remark 4. Choosing some special $\alpha_{j}$ and $d_{j}$ for $j=0,1, \ldots, n$, we can obtain some difference equations of the form $f(z)=\sum_{j=0}^{n} \alpha_{j} f\left(z+d_{j}\right)$. Thus, the main result is also valid for the discrete analytic functions (see [51-55]). For example, let

$$
\begin{gathered}
\frac{f(z+1+i)-f(z)}{i+1}=\frac{f(z+i)-f(z+1)}{i-1}, \\
\text { or } f(z)=i f(z+i)-i f(z-1)+f(z+1+i),
\end{gathered}
$$

which is the special case of (3). Thus, Theorem 2 is also valid for (10) or (11). When $z=m+i n$ and $m, n \in \mathbb{Z}$, equation (10) is the definition of discrete analytic and harmonic functions (see Hundhausen [56]). At this time, we can obtain the uniqueness of (10) or (11). Another definition of discrete analytic and harmonic functions:

$$
\begin{gathered}
f(z+1)-f(z)=\frac{f(z+i)-f(z)}{i}, \\
\text { or } f(z)=\frac{1-i}{2} f(z+1)+\frac{1+i}{2} f(z+i),
\end{gathered}
$$

can be seen in Harman [57]. Clearly, our theorem is also valid for (12) or (13).

We note that the main tool of proof for Theorem $1.2 \mathrm{in} \mathrm{Li}$ et al. [46] is the traditional Theorem 1.62 in [24]; however, it is invalid for Theorem 2 because $\sum_{j=0}^{n} \alpha_{j}$ can be nonzero. We specially thank Corollary 1.105 in [58] which will be our key tool and listed in Section 2 as Lemma 8. On the other hand, our methods and technologies are also different with Li et al. [46]; for example, the proof is divided into the six cases in Li et al. [46], but it is only two cases in the proof of Theorem 2 . For the other detail cases, please see the proof in Section 3. However, it is worth mentioning that the meromorphic function $f$ can be exactly expressed by

$$
f(z)=c \frac{1+e^{a z+b}}{1-e^{a z+b}}
$$

in some special cases. Certainly, some preliminaries of the difference value distribution theory [34-37] are also used in the proof of Theorem 2 .

In the final, the present paper will be organized as follows. In Section 2, we will give some preliminaries which can be seen in the listed references. And the main result will be proved in the final section.

\section{Some Lemmas}

In this paper, the value distribution theory established by R. Nevanlinna is the main tool for the studies. For convenience of the reader who might not be familiar with Nevanlinna theory, we list here some results from Nevanlinna theory (see, e.g., $[24,48])$.

The following known results are important in the value distribution theory (see, e.g., $[24,48]$ ).

(i) The arithmetic properties of $T(r, f)$ and $m(r, f)$ are as follows:

$$
\begin{array}{r}
T(r, f g) \leq T(r, f)+T(r, g), \\
T(r, f+g) \leq T(r, f)+T(r, g)+O(1) .
\end{array}
$$

The same inequalities hold for $m(r, f)$.

(ii) The Nevanlinna first fundamental theorem is as follows: $T(r, f)=T(r,(1 / f))+O(1)$.

(iii) The logarithmic derivative lemma is as follows: $m\left(r,\left(f^{\prime} / f\right)\right)=O(\log r)$, if the order $\rho(f)$ is finite. 
In the following, we present some lemmas, which will be needed in the sequel.

Lemma 1 (see Theorem 3 in [59]). Let $f$ and $g$ be two nonconstant rational functions. If $f$ and $g$ share $a_{1}, a_{2}, a_{3}, a_{4}$ $I M$, where $a_{1}, a_{2}, a_{3}, a_{4}$ are four distinct values in the extended complex plane, then $f=g$.

Lemma 2 (see Lemma 1 in [4]). Let $f$ and $g$ be two distinct nonconstant meromorphic functions, and let $a_{1}, a_{2}, a_{3}, a_{4}$ be four distinct values in the extended complex. If $f$ and $g$ share $a_{1}, a_{2}, a_{3}, a_{4} I M$, then for a set with finite linear measure $E$,

(i) $T(r, f)=T(r, g)+O(\log (r T(r, f)))$, as $r \notin E$ and $r \longrightarrow \infty$

(ii) $2 T(r, f)=\sum_{j=1}^{4} N\left(r, 1 /\left(f-a_{j}\right)\right)+O(\log (r T(r, f)))$, as as $r \notin E$ and $r \longrightarrow \infty$, where $N(r, 1)$ $(f-\infty))=N(r, f)$

Remark 5. Under the assumptions of Lemma 2, if $f$ and $g$ in Lemma 2 are of finite order, by the context of paper [26] and the proof of Lemma 1 in [26], we can find that the conclusion of Lemma 2 can be changed to

(i) $T(r, f)=T(r, g)+O(\log r)$, as $r \longrightarrow \infty$

(ii) $2 T(r, f)=\sum_{j=1}^{4} N\left(r, 1 /\left(f-a_{j}\right)\right) O(\log r)$, as $r \longrightarrow \infty$

Lemma 3 (see Lemma 2 and Corollary 1 in [3]). Let $f$ and $g$ be distinct nonconstant meromorphic functions that share four values $a_{1}, a_{2}, a_{3}$, and $a_{4} I M$, where $a_{4}=\infty$. Then, for $a$ set with finite linear measure $E$, the following statements hold:

(i) $N_{0}\left(r,\left(1 / f^{\prime}\right)\right)=O(\log (r T(r, f)))$ and $N_{0}\left(r,\left(1 / g^{\prime}\right)\right)=$ $O(\log (r T(r, f)))$ as $r \notin E, r \longrightarrow \infty$, and $E$ is a set with finite linear measure, where $N_{0}\left(r,\left(1 / f^{\prime}\right)\right)$ and $N_{0}\left(r,\left(1 / g^{\prime}\right)\right)$ count, respectively, only those points in $N\left(r,\left(1 / f^{\prime}\right)\right)$ and $N\left(r,\left(1 / f^{\prime}\right)\right)$ which do not occur when $f(z)=g(z)=a_{j}$ for some $j=1,2,3,4$.

(ii) For $j=1,2,3,4$, we next let $N_{2}\left(r, a_{j}\right)$ refer only to those $a_{j}$-points that are multiple for both $f$ and $g$ and count each such point the number of times of the smaller of the two multiplicities. Then, $\sum_{j=1}^{4} N_{2}\left(r, a_{j}\right)=$ $O(\log (r T(r, f)))$ as $r \notin E$ and $r \longrightarrow \infty$.

Remark 6. Under the assumptions of Lemma 3, if $f$ and $g$ in Lemma 3 are of finite order, by the context of paper [38] and the proof of Lemma 2 and Corollary 1 in [38], we can find that the conclusion of Lemma 3 can be changed into

(i) $N_{0}\left(r,\left(1 / f^{\prime}\right)\right)=O(\log r)$ and $N_{0}\left(r,\left(1 / g^{\prime}\right)\right)=O(\log r)$, as $r \longrightarrow \infty$

(ii) $\sum_{j=1}^{4} N_{2}\left(r, a_{j}\right)=O(\log r)$, as $r \longrightarrow \infty$

Lemma 4 (see Corollary 2.5 in [34]). Let $f$ be a nonconstant meromorphic function of finite order and $\eta$ be a fixed nonzero complex number. Then, for each $\varepsilon>0$,

$$
m\left(r, \frac{f(z+\eta)}{f(z)}\right)+m\left(r, \frac{f(z)}{f(z+\eta)}\right)=O\left(r^{\rho-1+\varepsilon}\right)
$$

$$
\text { as } r \longrightarrow \infty \text {. }
$$

Lemma 5 (see Theorem 1 in [4]). Let $f$ and $g$ be two nonconstant meromorphic functions that share $a_{1}, a_{2}, a_{3} I M$ and $a_{4} C M$, where $a_{1}, a_{2}, a_{3}$, and $a_{4}$ are four distinct values in the extended complex plane. Suppose that there exists some real constant $\mu>4 / 5$ and some set $I \subset(0,+\infty)$ that has infinite linear measure such that

$$
\frac{N\left(r,\left(1 /\left(f-a_{4}\right)\right)\right)}{T(r, f)} \geq \mu,
$$

for all $r \in I$, where $N\left(r,\left(1 /\left(f-a_{4}\right)\right)\right)=N(r, f)$, if $a_{4}=\infty$. Then, $f$ and $g$ share all four values CM.

Lemma 6 (see Theorem 4.3 in [24]). Let $f$ and $g$ be two distinct nonconstant meromorphic functions and $a_{j}(j=1,2,3,4)$ be four distinct values in the extend complex plane. If $f$ and $g$ share $a_{j}(j=1,2,3,4) \quad C M$, then $f(z)=T(g(z))$, where $T$ is a Mobius transformation such that two of the four values are fixed points and another two (are Picard exceptional values of $f$ and $g$ ) exchange each other under $T$.

Lemma 7 (see Theorem 1.62 in [24]). Let $f_{1}, f_{2}, \ldots, f_{n}$ be nonconstant meromorphic functions, and let $f_{n+1} \neq 0$ be a meromorphic function such that $\sum_{j=1}^{n+1} f_{j}=1$. Suppose that there exists a subset $I \subseteq R^{+}$whose linear measure is $\infty$ such that

$$
\sum_{i=1}^{n+1} N\left(r, \frac{1}{f_{i}}\right)+n \sum_{\substack{i=1 \\ i \neq j}}^{n+1} \bar{N}\left(r, f_{i}\right)<(\lambda+o(1)) T\left(r, f_{j}\right),
$$

$$
j=1,2, \ldots, n,
$$

As $r \in I$ and $r \longrightarrow \infty$, where $0 \leq \lambda<1$, then $f_{n+1} \equiv 1$.

Lemma 8 (see Corollary 1.105(iii) in [58]). Assume that entire functions $f_{0}, f_{1}, \ldots, f_{n}$ vanish nowhere on $\mathbb{C}^{m}$ such that

$$
f_{0}+f_{1}+\cdots+f_{n}=0 .
$$

Partition the index set $I=\{0,1, \ldots, n\}$ into subsets $I_{\alpha}$, $I=\cup_{\alpha=0}^{k} I_{\alpha}$, putting two indices $i$ and $j$ in the same subset $I_{\alpha}$ if and only if $\left(f_{i} / f_{j}\right)$ is a constant. Then, we have

$$
\sum_{i \in I_{\alpha}} f_{i}=0, \quad \alpha=0,1, \ldots, k .
$$

\section{Proof of Theorem 2}

If $L(z, f)$ is a constant, by the assumption that $f$ and $L(z, f)$ share $a_{1}, a_{2}, a_{3}$ IM and share $\infty \mathrm{CM}$, we know that $f$ has more than two Picard values. And it is impossible, so $L(z, f)$ 
is nonconstant. Assume that $f$ is a nonconstant rational function; according to the definition of $L(z, f)$, we can find that $L(z, f)$ is also a nonconstant rational function, and then using Lemma 1, we deduce that $L(z, f)=f$. Next, we suppose that $f$ is a nonconstant transcendental meromorphic function. Since $\rho(f)<\infty$ and $f$ and $L(z, f)$ share $a_{1}, a_{2}, a_{3}$ IM and share $\infty$ CM, applying Remark 5, we obtain

$$
T(r, f)=T(r, L(z, f))+S(r, f) .
$$

Then, we can see that $L(z, f)$ is also a transcendental meromorphic function. Next, we assume $L(z, f) \not \equiv f$.

Under the assumption that $f$ and $L(z, f)$ share $\infty \mathrm{CM}$, Remark 5 (ii) leads to

$$
N_{(2}(r, f)+N_{(2}(r, L(z, f))=2 N_{2}(r, \infty)=S(r, f),
$$

where $N_{(2}(r, f)$ denotes the counting function of the multiple poles of $f$ in $|z|<r$, and each point in $N_{(2}(r, f)$ is counted according to its multiplicity as a pole of $f$. $N_{(2}(r, L(z, f))$ is similar with $N_{(2}(r, f)$. Hence,

$$
N(r, L(z, f)-f) \leq \bar{N}_{(1,1)}(r, \infty)+S(r, f),
$$

where $\bar{N}_{(1,1)}(r, \infty)$ is the counting function of the common simple poles of $f$ and $g$. By the definition of $L(z, f)$ and Lemma 4, we deduce

$$
\begin{aligned}
m(r, L(z, f)-f) & =m\left(r, \frac{L(z, f)-f}{f} f\right) \\
& \leq m(r, f)+m\left(r, \frac{L(z, f)}{f}\right)+O(1) \\
& \leq m(r, f)+S(r, f) .
\end{aligned}
$$

From (23), (24), Remark 5 (ii), and the assumption that $f$ and $L(z, f)$ share $a_{1}, a_{2}, a_{3}$ IM and $\infty$ CM, we deduce

$$
\begin{aligned}
2 T(r, f) & =\bar{N}\left(r, \frac{1}{f-a_{1}}\right)+\bar{N}\left(r, \frac{1}{f-a_{2}}\right)+\bar{N}\left(r, \frac{1}{f-a_{3}}\right)+\bar{N}(r, f)+S(r, f) \\
& \leq \bar{N}\left(r, \frac{1}{L(z, f)-f}\right)+\bar{N}_{(1,1)}(r, \infty)+S(r, f) \\
& \leq T\left(r, \frac{1}{L(z, f)-f}\right)+\bar{N}_{(1,1)}(r, \infty)+S(r, f) \\
& =m(r, L(z, f)-f)+N(r, L(z, f)-f)+\bar{N}_{(1,1)}(r, \infty)+S(r, f) \\
& \leq m(r, L(z, f)-f)+2 \bar{N}_{(1,1)}(r, \infty)+S(r, f) \\
& \leq T(r, f)+\bar{N}_{(1,1)}(r, \infty)+S(r, f) .
\end{aligned}
$$

Hence, we get

$$
\lim _{z \in I} \frac{N(r, f)}{T(r, f)}=1,
$$

where $I \subset(0,+\infty)$ with logarithmic measure $\operatorname{logmes} I=+\infty$. In view of Lemma 5 , we deduce that $f$ and $L(z, f)$ share $a_{1}, a_{2}, a_{3}$ and $\infty$ CM. Then, by Lemma 6 , we obtain that $f$ is a Möbius transformation of $L(z, f)$. We set

$$
f=\frac{A L(z, f)+B}{C L(z, f)+D}
$$

where $A, B, C$, and $D$ are constants and $A D-B C \neq 0$. We consider the following two cases.

Case 1. Suppose that $C=0$. Then, from (27), we get

$$
f=A_{1} L(z, f)+B_{1},
$$

where $A_{1}(\neq 0)$ and $B_{1}$ are two constants, and $A_{1}=(A / D)$, and $B_{1}=(B / D)$. If two of $\left\{a_{1}, a_{2}, a_{3}\right\}$ are not the Picard exceptional value of $f$, we get $f \equiv L(z, f)$, which is a contradiction, so two of $\left\{a_{1}, a_{2}, a_{3}\right\}$ are Picard exceptional values of $f$. Without loss of generality, we set $a_{1}$ and $a_{2}$ as Picard exceptional values of $f$, and by the assumption of Theorem 2, we get $a_{1}$ and $a_{2}$ as Picard exceptional values of $L(z, f)$. By rewriting (28), we obtain

$$
f-a_{1}=A_{1}\left(L(z, f)-\frac{a_{1}-B_{1}}{A_{1}}\right),
$$

and noting $f \neq a_{1}$, we deduce $\left(\left(a_{1}-B_{1}\right) / A_{1}\right)=a_{1}$ or $a_{2}$. Similarly, we get

$$
f-a_{2}=A_{1}\left(L(z, f)-\frac{a_{2}-B_{1}}{A_{1}}\right),
$$

and $\left(\left(a_{2}-B_{1}\right) / A_{1}\right)=a_{1}$ or $a_{2}$.

$\left(\left(a_{1}-B_{1}\right) / A_{1}\right)=a_{1}$ and $\left(\left(a_{2}-B_{1}\right) / A_{1}\right)=a_{1}$ imply that $a_{1}=a_{2}$, which is impossible. By $\left(\left(a_{1}-B_{1}\right) / A_{1}\right)=a_{1}$ and $\left(\left(a_{2}-B_{1}\right) / A_{1}\right)=a_{2}$, we obtain $A_{1}=1$ and $B_{1}=0$, that is, $f \equiv L(z, f)$, which is a contradiction to the assumption. 
From $\left(\left(a_{1}-B_{1}\right) / A_{1}\right)=a_{2}$ and $\left(\left(a_{2}-B_{1}\right) / A_{1}\right)=a_{2}$, we have $a_{1}=a_{2}$ which is also impossible. Combining $\left(\left(a_{1}-B_{1}\right) /\right.$ $\left.A_{1}\right)=a_{2}$ and $\left(\left(a_{2}-B_{1}\right) / A_{1}\right)=a_{1}$, we get $A_{1}=-1$ and $B_{1}=a_{1}+a_{2}$, and then

$$
f+L(z, f)=a_{1}+a_{2} .
$$

Since $f(z) \neq a_{1}, a_{2}$, we set

$$
\frac{f-a_{1}}{f-a_{2}}=e^{\gamma(z)}
$$

where $\gamma(z)=a_{m} z^{m}+a_{m-1} z^{m-1}+\cdots+a_{1} z+a_{0}, a_{0}, a_{1}, \ldots$, $a_{m}(\neq 0)$, are constants, and $0<m \leq \rho(f)<\infty$. By a calculation, for $j=0,1,2, \ldots, n$, we deduce that

$$
\begin{aligned}
\gamma\left(z+d_{j}\right)= & \gamma(z)+a_{m} C_{m}^{1} d_{j} z^{m-1} \\
& +\left(a_{m} C_{m}^{1} d_{j}^{2}+a_{m-1} C_{m-1}^{1} d_{j}\right) z^{m-2}+\cdots .
\end{aligned}
$$
obtain

Set $a_{m}=\left|a_{m}\right| e^{i \theta_{m}}$; then, when $z=r e^{-i\left(\theta_{m} / m\right)} \longrightarrow \infty$, we

$$
\begin{aligned}
\operatorname{Re} \gamma(z) & \sim\left|a_{m}\right| r^{m}, \\
e^{\gamma(z)} & \longrightarrow \infty,
\end{aligned}
$$

and while $z=r e^{-i\left(\theta_{m} / m\right)+(\pi / m)} \longrightarrow \infty$, we know

$$
\begin{aligned}
\operatorname{Re} \gamma(z) & \sim-\left|a_{m}\right| r^{m}, \\
e^{\gamma(z)} & \longrightarrow 0 .
\end{aligned}
$$

Equation (32) implies

$$
f=\frac{a_{1}-a_{2} e^{\gamma(z)}}{1-e^{\gamma(z)}} .
$$

So,

$$
L(z, f)=\sum_{j=0}^{n} \alpha_{j} \frac{a_{1}-a_{2} e^{\gamma\left(z+d_{j}\right)}}{1-e^{\gamma\left(z+d_{j}\right)}} .
$$

Substituting (36) and (37) to (31), we get

$$
\frac{a_{1}-a_{2} e^{\gamma(z)}}{1-e^{\gamma(z)}}+\sum_{j=0}^{n} \alpha_{j} \frac{a_{1}-a_{2} e^{\gamma\left(z+d_{j}\right)}}{1-e^{\gamma\left(z+d_{j}\right)}}=a_{1}+a_{2} .
$$

When $z=r e^{-i\left(\theta_{m} / m\right)} \longrightarrow \infty$, from (33), (34), and (38), we know

$$
a_{2}+a_{2} \sum_{j=0}^{n} \alpha_{j}=a_{1}+a_{2},
$$

$$
a_{1}+a_{1} \sum_{j=0}^{n} \alpha_{j}=a_{1}+a_{2}
$$

Combining (39) and (40), we deduce that

$$
\begin{aligned}
\sum_{j=0}^{n} \alpha_{j}+1 & =0, \\
a_{2} & =-a_{1}, \\
B_{1} & =0, \\
a_{3} & =0 .
\end{aligned}
$$

Then, by (31), we obtain

$$
f+L(z, f)=0 .
$$

Substituting (41) to (36) and (37), we have

$$
f=a_{1} \frac{1+e^{\gamma(z)}}{1-e^{\gamma(z)}}
$$

$$
L(z, f)=a_{1} \sum_{j=0}^{n} \alpha_{j} \frac{1+e^{\gamma\left(z+d_{j}\right)}}{1-e^{\gamma\left(z+d_{j}\right)}},
$$

$$
\sum_{j=0}^{n} \alpha_{j}=-1
$$

Substituting (43) and (44) to (42), we deduce

$$
\frac{1+e^{\gamma(z)}}{1-e^{\gamma(z)}}+\sum_{j=0}^{n} \alpha_{j} \frac{1+e^{\gamma\left(z+d_{j}\right)}}{1-e^{\gamma\left(z+d_{j}\right)}}=0 .
$$

Denoting $\beta_{n+1}=1, d_{n+1}=0, \quad$ and $\beta_{j}=\alpha_{j}$, where $j=0,1, \ldots, n$, then

$$
\sum_{j=0}^{n+1} \beta_{j} \frac{1+e^{\gamma\left(z+d_{j}\right)}}{1-e^{\gamma\left(z+d_{j}\right)}}=0
$$

where $\sum_{j=0}^{n+1} \beta_{j}=0$. If there exist some $j, 0 \leq j \leq n$, such that $d_{j}=0$, we denote $\beta_{n+1}=1+d_{j}$ and $\beta_{j}=0$; then, we get a similar equation with the above one.

So,

$$
\sum_{j=0}^{n+1} \beta_{j}\left(1+e^{\gamma\left(z+d_{j}\right)}\right) \prod_{i \neq j}\left(1-e^{\gamma\left(z+d_{j}\right)}\right)=0 .
$$

Then, by a calculation, we get

and when $z=r e^{-i\left(\theta_{m} / m\right)+(\pi / m)} \longrightarrow \infty$, we get

$$
\sum_{j=0}^{n+1} \beta_{j} e^{\gamma\left(z+d_{j}\right)}+\sum_{k=2}^{n}(-1)^{k-1} \sum_{i_{1}, i_{2}, \ldots, i_{k}}\left(\beta_{i_{1}}+\cdots+\beta_{i_{k}}\right) e^{\gamma\left(z+d_{i_{1}}\right)+\cdots+\gamma\left(z+d_{i_{k}}\right)}=0,
$$


where $\Sigma_{i_{1}, i_{2}, \ldots, i_{k}}$ denotes the sum for any $k$ numbers in $\{0,1, \ldots, n+1\}$. Applying Lemma 8 , we get that there exits nonnegative integer $i, j$ and $i \neq j$ such that $\gamma\left(z+d_{i}\right)-\gamma(z+$ $d_{j}$ ) is a constant. From (33), we know that $\gamma(z)$ is a polynomial of $\operatorname{deg} \gamma=1$. We set

$$
\gamma(z)=a z+b
$$

where $a \neq 0, b$ are constants. We substitute into (43) and get

$$
f(z)=a_{1} \frac{1+e^{a z+b}}{1-e^{a z+b}}
$$

where $a \neq 0, b$ are constants.

Case 2. Suppose that $C \neq 0$. By (27), we get

$$
f=\frac{A_{1} L(z, f)+B_{1}}{L(z, f)+D_{1}},
$$

where $\quad A_{1}=(A / C), \quad B_{1}=(B / C), \quad D_{1}=(D / C)$, and $A_{1} D_{1}-B_{1} \neq 0$.

Since $f$ and $L(z, f)$ share $\infty$ CM, from (52), we know $\infty$ is a Picard exceptional value of $f$ and $L(z, f)$. If $f$ can take every one of $\left\{a_{1}, a_{2}, a_{3}\right\}$, we get at least two of $\left\{a_{1}, a_{2}, a_{3}\right\}$ are equal, which is a contradiction, so one of $\left\{a_{1}, a_{2}, a_{3}\right\}$ is a Picard exceptional value of $f$ and $L(z, f)$. Without loss of generality, we set $a_{3}$ is a Picard exceptional value of $f$; by assumption, $a_{3}$ is also a Picard exceptional value of $L(z, f)$. Noting that $\infty$ is Picard exceptional values of $L(z, f)$ and they share $\infty \mathrm{CM}$, by (52), we have $A_{1}$ is a Picard exceptional value of $f$, so $A_{1}=a_{3}$. Since $\infty$ is Picard exceptional values of $f$ and they share $\infty \mathrm{CM}$; by (52), we see that $-D_{1}$ is a Picard exceptional value of $L(z, f)$. Then, we have $-D_{1}=a_{3}$. Thus, (52) turns to be

$$
f=\frac{a_{3} L(z, f)+B_{1}}{L(z, f)-a_{3}}
$$

where $a_{3}$ and $B_{1}$ are not all zero.

Noting that $f$ and $L(z, f)$ share $a_{1}$ and $a_{2}$ CM and neither of them is a Picard exceptional value of $f$ and $L(z, f)$, from (53), we get

$$
\begin{aligned}
& a_{1}=\frac{a_{3} a_{1}+B_{1}}{a_{1}-a_{3}}, \\
& a_{2}=\frac{a_{3} a_{2}+B_{1}}{a_{2}-a_{3}} .
\end{aligned}
$$
have

So, $a_{3}=\left(\left(a_{1}+a_{2}\right) / 2\right)$ and $B_{1}=-a_{1} a_{2}$. Then, by (53), we

$$
f=\frac{\left(\left(a_{1}+a_{2}\right) / 2\right) L(z, f)-a_{1} a_{2}}{L(z, f)-\left(\left(a_{1}+a_{2}\right) / 2\right)} .
$$

By rewriting it, we obtain

$$
f L(z, f)-\frac{a_{1}+a_{2}}{2} f-\frac{a_{1}+a_{2}}{2} L(z, f)=-a_{1} a_{2} .
$$

Noting that both $\infty$ and $a_{3}$ are Picard exceptional values of $f$ and $L(z, f)$ and $a_{3}=\left(\left(a_{1}+a_{2}\right) / 2\right)$, we set

$$
f=\frac{a_{1}+a_{2}}{2}+e^{\beta(z)} \text {, }
$$

where $\beta(z)=b_{p} z^{p}+b_{p-1} z^{p-1}+\cdots+b_{1} z+b_{0}, \quad b_{0}, b_{1}, \ldots$, $b_{p}(\neq 0)$, are constants, and $p=\rho(f)>0$. Then, by the definition of $L(z, f)$, we get

$$
\begin{aligned}
L(z, f)= & \sum_{j=0}^{n} \alpha_{j} f\left(z+d_{j}\right) \\
= & \sum_{j=0}^{n} \alpha_{j}\left(\frac{a_{1}+a_{2}}{2}+e^{\beta\left(z+d_{j}\right)}\right) \\
= & \alpha_{0}\left(\frac{a_{1}+a_{2}}{2}+e^{\beta\left(z+d_{0}\right)}\right)+\alpha_{1}\left(\frac{a_{1}+a_{2}}{2}+e^{\beta\left(z+d_{1}\right)}\right) \\
& +\cdots+\alpha_{n}\left(\frac{a_{1}+a_{2}}{2}+e^{\beta\left(z+d_{n}\right)}\right) .
\end{aligned}
$$

Substituting (57) and (58) to the above equation, we have

$$
\left(\alpha_{0}+\alpha_{1}+\cdots+\alpha_{n}-1\right) \frac{a_{1}+a_{2}}{2} e^{\beta(z)}+\alpha_{0} e^{\beta\left(z+d_{0}\right)+\beta(z)}+\alpha_{1} e^{\beta\left(z+d_{1}\right)+\beta(z)}+\cdots+\alpha_{n} e^{\beta\left(z+d_{n}\right)+\beta(z)}=\frac{\left(a_{1}-a_{2}\right)^{2}}{4}
$$

Then, using Lemma 7, we get a contradiction. Thus, we complete the proof of Theorem 2 .

\section{Data Availability}

The data used to support the findings of this study are included within the article.

\section{Conflicts of Interest}

The authors declare that there are no conflicts of interest regarding the publication of this paper.

\section{Acknowledgments}

The study was supported by the National Natural Science Foundation of China (Grant no. 11871314) and the Scientific Research Project of Shanxi Datong University (Grant no. 2020K19).

\section{References}

[1] R. Nevanlinna, The Picard-Borel Theorem and the Theory of Meromorphic Functions, Reprinting of the 1929 Original, p. 171, Chelsea Publishing Co., New York, NY, USA, 1974. 
[2] G. G. Gundersen, "Meromorphic functions that share three or four values," Journal of the London Mathematical Society, vol. s2-20, no. 3, pp. 457-466, 1979.

[3] G. G. Gundersen, "Meromorphic functions that share four values," Transactions of the American Mathematical Society, vol. 277, no. 2, p. 545, 1983.

[4] G. G. Gundersen, "Meromorphic functions that share three values IM and a fourth value CM," Complex Variables and Elliptic Equations, vol. 20, no. 1-4, pp. 99-106, 1992.

[5] E. Mues, "Remarks on the four-point theorem," Complex Methods on Partial Differential Equations, Mathematical Research, vol. 53, pp. 109-117, Akademie-Verlag, Berlin, Germany, 1989.

[6] E. Mues, "Meromorphic functions sharing four values," Complex Variables and Elliptic Equations, vol. 12, no. 1-4, pp. 169-179, 1989.

[7] E. Mues, "Shared value problems for meromorphic functions," Value Distribution Theory and Complex Differential Equations, Joensuu, 1994, 17C43, Joensuun Yliop. Luonnont. Julk., Vol. 35University of Joensuu Publications in Sciences, Joensuu, Finland, 1995.

[8] M. Reinders, "Uniqueness theorems for meromorphic functions sharing four values," News from the Mathematical Seminar Giessen, vol. 200, pp. 15-38, 1991.

[9] M. Reinders, "A new example of meromorphic functions sharing four values and a uniqueness theorem," Complex Variables and Elliptic Equations, vol. 18, no. 3, pp. 213-221, 1992.

[10] S. P. Wang, "On meromorphic functions that share four values," Journal of Mathematical Analysis and Applications, vol. 173, no. 2, pp. 359-369, 1993.

[11] H. Ueda, "Some estimates for meromorphic functions sharing four values," Kodai Mathematical Journal, vol. 17, no. 2, pp. 329-340, 1994.

[12] H. Ueda, "Meromorphic functions $f$ and g that share two values CM and two values in the sense of $E_{k}(\beta, f)=E_{k}(\beta, \mathrm{g})$," Kodai Mathematical Journal, vol. 21, no. 3, pp. 273-284, 1998.

[13] H. X. Yi and C. T. Zhou, "Meromorphic functions that share four values," Journal of Shandong University, vol. 31, no. 2, pp. 121-128, 1996.

[14] T. P. Czubiak and G. G. Gundersen, "Meromorphic functions that share pairs of values," Complex Variables and Elliptic Equations, vol. 34, no. 1-2, pp. 35-46, 1997.

[15] G. Qiu, "Four values theorem of derivatives of meromorphic functions," Journal of Systems Science and Mathematical Sciences, vol. 11, no. 3, pp. 245-248, 1998.

[16] J. P. Wang, "Meromorphic functions sharing four values," Indian Journal of Pure and Appled Mathematics, vol. 32, no. 1, pp. 37-46, 2001.

[17] J. P. Wang, "On Nevanlinna's four-value theorem," Southeast Asian Bulletin of Mathematics, vol. 29, no. 1, pp. 169-178, 2005.

[18] B. Huang, "Some results on Gunderden's problem," Journal of Systems Science and Mathematical Sciences, vol. 23, no. 4, pp. 467-481, 2003.

[19] B. Huang, "On the unicity of meromorphic functions that share four values," Indian Journal of Pure and Applied Mathematics, vol. 35, no. 3, pp. 359-372, 2004.

[20] B. Huang and J. Du, "Meromorphic functions that share four values," Acta Mathematica Scientia, vol. 24, no. 4, pp. 529-535, 2004.

[21] K. Ishizaki, "Uniqueness problems on meromorphic functions which share four small functions," Proceedings of the Second ISAAC Congress, pp. 467-472, 2000.
[22] P. Li, "Meromorphic functions that share four small functions," Journal of Mathematical Analysis and Applications, vol. 263, no. 1, pp. 316-326, 2001.

[23] W. H. Yao, "Meromorphic functions sharing four small functions on 2CM+2IM," Archive of Mathematics, vol. 81, pp. 35-54, 2004.

[24] C. Yang and H. Yi, Uniqueness Theory of Meromorphic Functions, Kluwer Academic Publishers Group, Dordrecht, Netherlands, 2003.

[25] W. C. Lin and K. Ishizaki, "A "3IM+1CM" result for periodic meromorphic functions," Journal of Mathematical Analysis and Applications, vol. 466, no. 1, pp. 26-732, 2018.

[26] A. Banerjee and M. Ahamed, "On some sufficient conditions for periodicity of meromorphic function under new shared sets," Filomat, vol. 33, no. 18, pp. 6055-6072, 2019.

[27] I. Lahiri and K. Sinha, "Linear differential polynomials sharing a set of the roots of unity," Communications of the Korean Mathematical Society, vol. 35, no. 3, pp. 773-787, 2020.

[28] G. G. Gundersen, "Meromorphic functions that share finite values with their derivative," Journal of Mathematical Analysis and Applications, vol. 75, no. 2, pp. 441-446, 1980.

[29] G. Gundersen, "Meromorphic functions that share two finite values with their derivative," Pacific Journal of Mathematics, vol. 105, no. 2, pp. 299-309, 1983.

[30] E. Mues and N. Steinmetz, "Meromorphic functions that share values with their derivation," Manuscripta Mathmatica, vol. 29, no. 2-4, pp. 195-206, 1979.

[31] E. Mues and N. Steinmetz, "Meromorphic functions that share two values with their derivative," Results in Mathematics, vol. 6, no. 1-2, pp. 48-55, 1983.

[32] E. Mues and M. Reinders, "Meromorphic functions that share three values with a linear differential polynomial," Results in Mathematics, vol. 22, no. 3-4, pp. 725-738, 1992.

[33] G. Frank and X. H. Hua, "Differential polynomials that share three values IM with their generated meromorphic function," Michigan Mathematical Journal, vol. 46, no. 1, pp. 175-186, 1999.

[34] Y.-M. Chiang and S.-J. Feng, "On the Nevanlinna characteristic of $f(z+\eta)$ and difference equations in the complex plane," The Ramanujan Journal, vol. 16, no. 1, pp. 105-129, 2008.

[35] R. G. Halburd and R. J. Korhonen, "Nevanlinna theory for the difference operator," Annales Academice Scientiarum Fennicae Mathematica, vol. 31, no. 2, pp. 463-478, 2006.

[36] R. G. Halburd and R. J. Korhonen, "Difference analogue of the lemma on the logarithmic derivative with applications to difference equations," Journal of Mathematical Analysis and Applications, vol. 314, no. 2, pp. 477-487, 2006.

[37] I. Laine and C.-C. Yang, "Clunie theorems for difference and q -difference polynomials," Journal of the London Mathematical Society, vol. 76, no. 3, pp. 556-566, 2007.

[38] A. Banerjee and S. Bhattacharyya, "Uniqueness of meromorphic functions with their reduced linear c-shift operators sharing two or more values or sets," Advances in Difference Equations, vol. 509, pp. 1-23, 2019.

[39] M. B. Ahamed, "An investigation on the conjecture of Chen and Yi," Results in Mathematics, vol. 74, no. 122, pp. 1-28, 2019.

[40] M. B. Ahamed, "Correction to: an investigation on the conjecture of Chen and Yi," Results in Mathematics, vol. 74, no. 143, pp. 1-2, 2019.

[41] L. Ma, D. Liu, D. Liu, and M. Fang, "Uniqueness of meromorphic functions concerning sharing two small functions 
with their derivatives," Journal of Applied Analysis \& Computation, vol. 10, no. 2, pp. 713-728, 2020.

[42] Y. Y. Jiang, L. Liao, Z. X. Li Liao, and Z. Chen, "The value distribution of meromorphic solutions of some second order nonlinear difference equation," Journal of Applied Analysis \& Computation, vol. 8, no. 1, pp. 32-41, 2018.

[43] K. S. Charak, R. J. Korhonen, and G. Kumar, "A note on partial sharing of values of meromorphic functions with their shifts," Journal of Mathematical Analysis and Applications, vol. 435, no. 2, pp. 1241-1248, 2016.

[44] W. C. Lin, X. Q. Lin, and A. D. Wu, "Meromorphic functions partially shared values with their shifts," Bulletin of the Korean Mathematical Society, vol. 55, no. 2, pp. 469-478, 2018.

[45] W. C. Lin and K. Tohge, "Periodicity of meromorphic functions and partial sharing values," Houston Journal of Mathematics, vol. 46, no. 3, pp. 583-610, 2020.

[46] X.-M. Li, Y. Liu, and H.-X. Yi, "Meromorphic functions sharing four values with their difference operators," Computational Methods and Function Theory, vol. 21, no. 2, pp. 317-341, 2021.

[47] X.-M. Li and H.-X. Yi, "Meromorphic functions sharing four values with their difference operators or shifts," Bulletin of the Korean Mathematical Society, vol. 53, no. 4, pp. 1213-1235, 2016.

[48] W. K. Hayman, Meromorphic Functions, Oxford Mathematical Monographs, Clarendon Press, Oxford, UK, 1964.

[49] N. Steinmetz, "A uniqueness theorem for three meromorphic functions," Annales Academiae Scientiarum Fennicae. Series A. I. Mathematica, vol. 13, no. 1, pp. 93-110, 1988.

[50] J. Heittokangas, R. J. Korhonen, I. Laine, and J. Rieppo, "Uniqueness of meromorphic functions sharing values with their shifts," Complex Variables and Elliptic Equations, vol. 56, no. 1-4, pp. 81-92, 2011.

[51] I. Benjamini and O. Schramm, "Harmonic functions on planar and almost planar graphs and manifolds, via circle packings," Inventiones Mathematicae, vol. 126, no. 3, pp. 565-587, 1996.

[52] R. L. Brooks, C. A. B. Smith, A. H. Stone, and W. T. Tutte, "The dissection of rectangles into squares," Duke Mathematical Journal, vol. 7, no. 1-2, pp. 312-340, 1940.

[53] R. J. Duffin, "Basic properties of discrete analytic functions," Duke Mathematical Journal, vol. 23, no. 2, pp. 335-363, 1956.

[54] J. Ferrand, "Preharmonic functions and preholomorphic functions," Bulletin des Sciences Mathematiques, vol. 68, no. 2, pp. 152-180, 1944.

[55] C. Mercat, "Discrete Riemann surfaces and the Ising model," Communications in Mathematical Physics, vol. 218, no. 1, pp. 177-216, 2001.

[56] J. R. Hundhausen, "A generalization of discrete analytic and harmonic functions," Journal of Mathematical Analysis and Applications, vol. 25, no. 3, pp. 628-652, 1969.

[57] C. J. Harman, "A new definition of discrete analytic functions," Bulletin of the Australian Mathematical Society, vol. 10, no. 2, pp. 281-291, 1974.

[58] P. Hu, P. Li, and C. Yang, Unicity of Meromorphic Mappings, Kluwer Academic Publishers, Dordrecht, Netherlands, 2003.

[59] W. W. Adams and E. G. Straus, "Non-Archimedian analytic functions taking the same values at the same points," Illinois Journal of Mathematics, vol. 15, no. 3, pp. 418-424, 1971. 